\title{
How to avoid "en masse" lobectomy using VATS technique in presence of hilar fibrosis
}

\author{
Dario Amore', Dino Casazza ${ }^{1}$, Alessandro Saglia ${ }^{2}$, Pasquale Imitazione ${ }^{2}$, Umberto Caterino ${ }^{2}$, Carlo Curcio ${ }^{1}$ \\ ${ }^{1}$ Department of Thoracic Surgery, Monaldi Hospital, Naples; ${ }^{2}$ Department of Respiratory Diseases, Monaldi Hospital, \\ Naples, Italy
}

\begin{abstract}
Perivascular fibrosis is technically one of the most challenging issue to manage during thoracoscopic lobectomy and it is associated with increased risk of hemorrhagic injury. Here we report a case of thoracoscopic right lower lobectomy performed with individual dissection of segmental arteries due to dense adventitial fibrosis around the right lower lobe pulmonary artery. This approach may be considered as an alternative to the so-called "en masse" lobectomy and a way to avoid conversion to thoracotomy.
\end{abstract}

\section{Introduction}

In the last decades, video-assisted thoracic surgery (VATS) has been widely adopted by many thoracic surgeons for the treatment

Correspondence: Dario Amore, Monaldi Hospital, Via Leonardo Bianchi 1, 80131 Naples, Italy.

E-mail: dario.amore@alice.it

Keywords: Lung cancer; VATS lobectomy; vascular fibrosis.

Contributions: All the authors contributed equally. All the authors have read and approved the final version of the manuscript and agreed to be accountable for all aspects of the work.

Conflict of interest: the authors declare that they have no competing interests.

Ethics approval: No ethical committee approval was required for this case report by the Department, because this article does not contain any studies with human participants or animals. Informed consent was obtained from the patient included in this study.

Consent for publication: The patient gave his written consent to use his personal data for the publication of this case report and any accompanying images.

Received for publication: 5 October 2020.

Accepted for publication: 17 January 2021.

${ }^{\circ}$ Copyright: the Author(s), 2021

Licensee PAGEPress, Italy

Monaldi Archives for Chest Disease 2021; 91:1633

doi: 10.4081/monaldi.2021.1633

This article is distributed under the terms of the Creative Commons Attribution Noncommercial License (by-nc 4.0) which permits any noncommercial use, distribution, and reproduction in any medium, provided the original author(s) and source are credited. of early-stage lung cancer, showing better outcomes in the immediate postoperative period than open surgery. Overcoming the initial learning curve, high volume thoracic surgical centers began to perform complex cases of VATS lobectomy due to chemo-radiotherapy, centrally located tumors, dense hilar lymphadenopathy, etc. [1]. Perivascular and peribronchial fibrosis are one of the most complex scenarios encountered during major lung resection in minimally invasive surgery and may require, in such instances, simultaneous stapling of lobar bronchus and pulmonary artery or vein [2]. Here we present an alternative approach to perform VATS lobectomy in presence of dense fibrous tissue around bronchovascular structures.

\section{Case Report}

A 68-year-old man was admitted to our hospital for surgical treatment of pulmonary adenocarcinoma located in the right lower lobe (Figure 1A) and classified as clinical T1cN0M0 according to the $8^{\text {th }}$ edition of the TNM classification for non-small lung cancer. He underwent video-assisted thoracoscopic right lower lobectomy and lymph node dissection using a standardized three-port anterior approach. During the procedure we found a perivascular fibrosis around the right lower lobe pulmonary artery (Figure 1B) with no apparent cleavage plane between inferior lobar artery and right lower lobe bronchus. After transection of the inferior pulmonary vein, the basal segmental arteries were exposed in the fissure using a radiofrequency-based device, dissected separately with a rightangle clamp and individually divided with a vascular endostapler (Figure 2). The lobectomy was then completed by staple-division of the right lower lobe bronchus plus a single superior segmental artery and performing hilar-mediastinal lymph node dissection. The patient had an uneventful recovery and was discharged home on the fourth postoperative day. Pathological stage was IA3.

\section{Discussion}

When hilar vessels and bronchi are involved by dense fibrous adhesions, the individual dissection of the bronchovascular structures during VATS lobectomy can be so difficult to require planned or emergency conversion to thoracotomy. In such instance, some authors propose the so-called "en mass" or "en bloc" lobectomy which consist of a bulk hilar division with stapler [3]. The international community of thoracic surgeons expressed doubts regarding the safety of this technique due to potential adverse events such as intraoperative bleeding and 


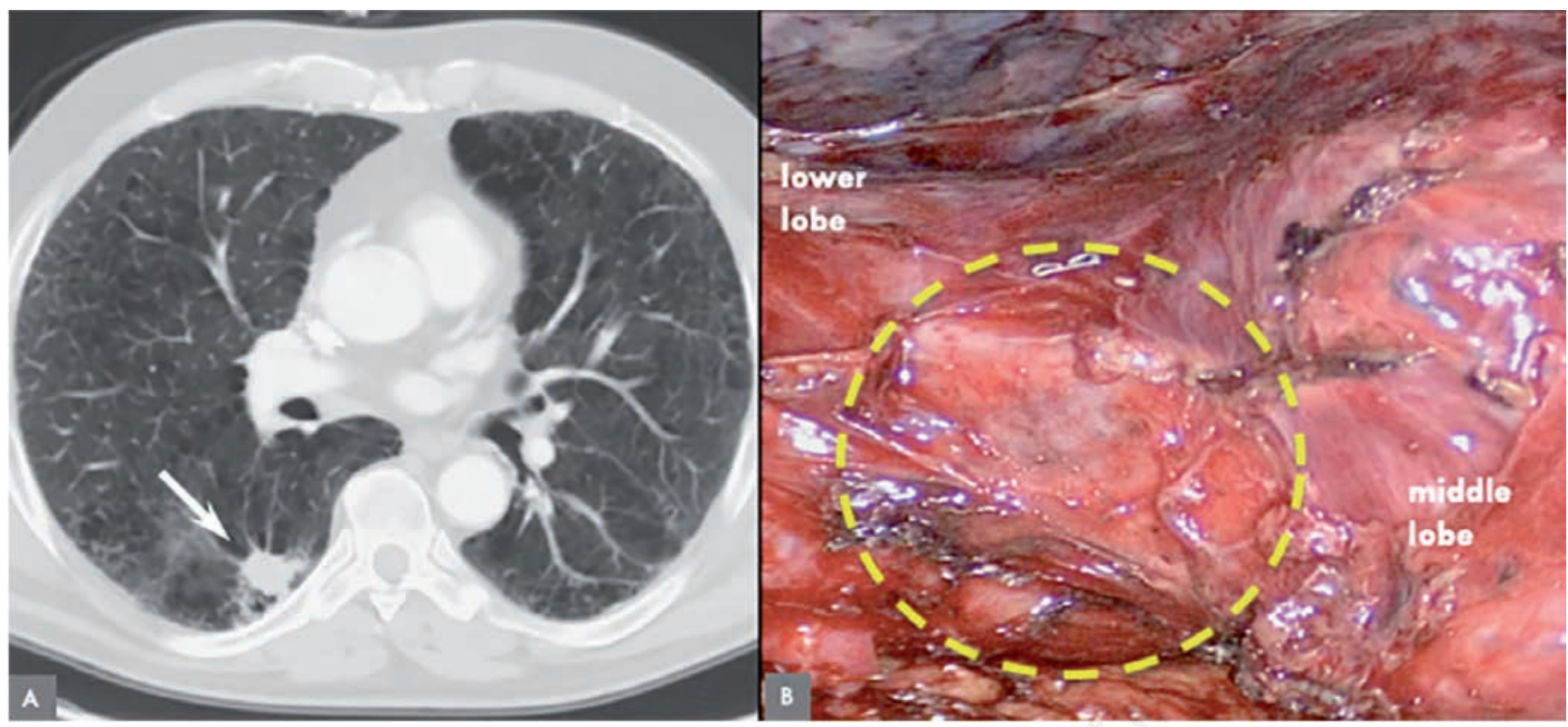

Figure 1. A) Chest computed tomography (CT) scan showing lung cancer in the right lower lobe (white arrow). B) Adventitial fibrosis of the right lower lobe artery (dashed yellow circle highlights the area) encountered during the dissection of the major fissure.

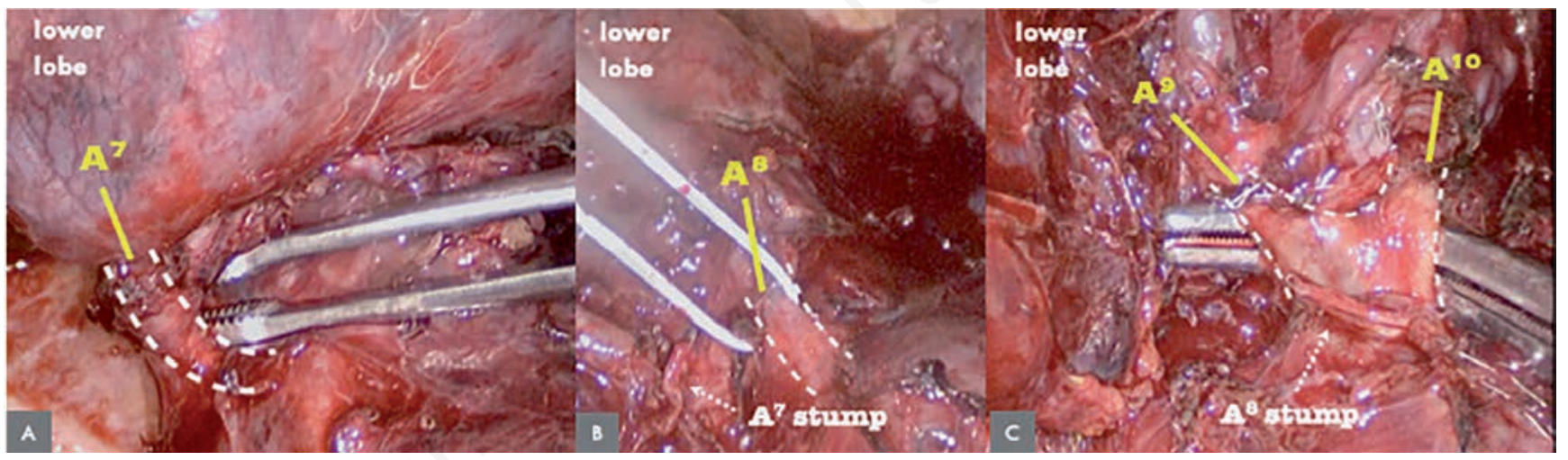

Figure 2. Intraoperative view of basal segmental arteries of the right lower lobe individually dissected. A7, medial basal segmental artery; A8, anterior basal segmental artery; A9, lateral basal segmental artery; A10, posterior basal segmental artery.

bronchopleural fistula, even if there is currently no report of such complications resulting from simultaneous stapling of hilum. However, some authors, for prevention of hemorrhagic complications during simultaneous stapling of bronchovascular structures, suggest selecting the appropriate type of stapler and to clamp the target at the thinnest part, proceeding with more caution in the presence of hilar lesions or calcified hilar lymph nodes [4]. The question of the bronchopleural or bronchovascular fistula remains unresolved and very controversial. Some authors argue that such complication may be increased, compared to the individual dissection of bronchovascular structures, firing an infected bronchus and pulmonary artery together by simultaneous stapling of hilum [3]. On the other hand, cases of arteriobronchial fistula have been reported in the literature even after VATS lobectomy performed by individual division of hilar structures $[5,6]$ and despite various techniques have been reported to reinforce the bronchial stump after lobectomy, such as use of pleural, pericardial and muscle flaps, the method yielding the best results still remains unclear [7]. Our case, through the individual dissection and closure of segmental arteries, shows how to avoid simultaneous bronchovascular stapling or conversion to thoracotomy when severe fibrous adhesions are encountered between the pulmonary artery and bronchus during VATS lobectomy.

\section{References}

1. Pischik VG. Technical difficulties and extending the indications for VATS lobectomy. J Thorac Dis 2004;6:S623-30. 
2. Murakami J, Ueda K, Hayashi M, et al. Simultaneous stapling of the lobar bronchus and pulmonary artery: is it actually dangerous? Interact Cardiovasc Thorac Surg 2016;22:671-3.

3. Qiang G, Nakajima J. Simultaneous stapling of pulmonary vein and bronchus in video-assisted thoracic surgery lobectomy. Ann Thorac Cardiovasc Surg 2015;21:78-80.

4. Kamiyoshihara M, Igai $\mathrm{H}$, Ibe $\mathrm{T}$, et al. Pulmonary lobar root clamping and stapling technique: return of the "en masse lobectomy". Gen Thorac Cardiovasc Surg 2013;61:280-91.
5. Marjanski T, Sternau A, Dziedzic R. Arteriobronchial fistula complicating right upper lobectomy. Ann Thorac Surg 2013; 96:e95-6.

6. Abe J, Hasumi T, Takahashi S, et al. Fatal broncho-pulmonary artery fistula after lobectomy for lung cancer. J Surg Case Rep 2015;9:1-2.

7. Matsuoka K, Imanishi N, Yamada T, et al. Clinical results of bronchial stump coverage using free pericardial fat pad. Interact Cardiovasc Thorac Surg 2016;23:553-9. 